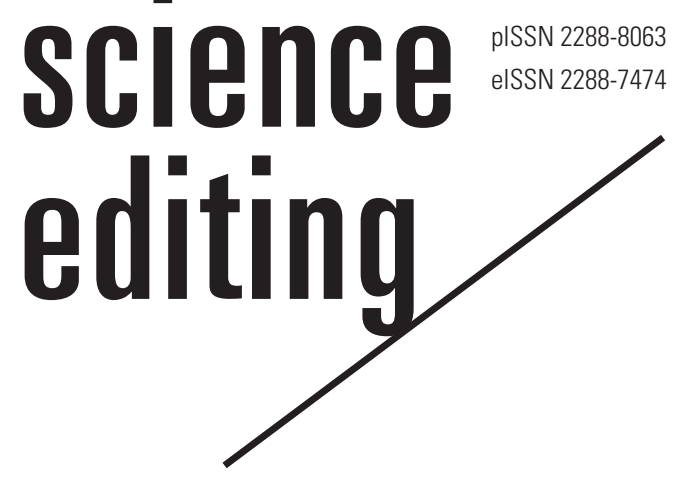

\title{
Changes in article share and growth by publisher and access type in Journal Citation Reports 2016, 2018, and 2020
}

\author{
Sang-Jun Kim', Kay Sook Park ${ }^{2}$ \\ Library, ${ }^{1}$ Korea Research Institute of Bioscience and Biotechnology, ${ }^{2}$ Electronics and Telecommunications Research Institute,
} Daejeon, Korea

\section{Abstract}

Purpose: This study explored changes in the journal publishing market by publisher and access type using the major journals that publish about $95 \%$ of Journal Citation Reports (JCR) articles.

Methods: From JCR 2016, 2018, and 2020, a unique journal list by publisher was created in Excel and used to analyze the compound annual growth rate by pivot tables. In total, 10,953 major JCR journals were analyzed, focusing on publisher type, open access (OA) status, and mega journals (publishing over 1,000 articles per year).

Results: Among the 19 publishers that published over 10,000 articles per year, in JCR 2020, six large publishers published $59.6 \%$ of the articles and 13 publishers $22.5 \%$. The other publishers published $17.9 \%$. Large and OA publishers increased their article share through leading mega journals, but the remaining publishers showed the opposite tendency. In JCR 2020, mega journals had a $26.5 \%$ article share and an excellent distribution in terms of the Journal Impact Factor quartile. Despite the high growth $(22.6 \%)$ and share $(26.0 \%)$ of OA articles, the natural growth of non-OA articles (7.3\%) and total articles (10.7\%) caused a rise in journal subscription fees. Articles, citations, the impact factor, and the immediacy index all increased gradually, and the compound annual growth rate of the average immediacy index was almost double than that of the average impact factor in JCR 2020.

Conclusion: The influence of OA publishers has grown under the dominance of large publishers, and mega journals may substantially change the journal market. Journal stakeholders should pay attention to these changes.

Received: September 27, 2021 Accepted: November 9, 2021

Correspondence to Sang-Jun Kim sjkim@kribb.re.kr

ORCID

Sang-Jun Kim

https://orcid.org/0000-0002-1833-2236 Kay Sook Park

https://orcid.org/0000-0002-2771-4930
Keywords

Journal Citation Reports; Journal publisher; Major journal; Mega journal; Open access

\section{Introduction}

Background/rationale: Over the past 20 years since the advent of e-journals, there have been many changes in the journal publishing environment, including an increase in new journals, 
the frequent extinction of small publishers due to mergers and acquisitions, a strengthened position of large commercial publishers, and the pursuit of open access (OA) opposed to the subscription model. The emergence of OA publishers was associated with a new publishing business model, substituting article processing charges (APCs) for subscription fees. Traditional print journal publishers, which had long monopolized libraries throughout the world, no longer overlook their competition with OA publishers. With the increase of OA journals and articles, the journal publishing environment continues to undergo unprecedented changes. Therefore, all stakeholders of academic journals need to pay close attention to changes and trends in the journal publishing environment.

Comparing the publication trends of the journals included in major bibliographic databases by year is effective because they cover the world's major journals and publishers. Although some studies have dealt with changes in journal publishing in a fragmentary manner, such as OA publishing based on Web of Science (WoS), Journal Citation Reports (JCR), Directory of Open Access Journals (DOAJ), and Scopus, it is difficult to find accurate and up-to-date research on major publishers focusing on core journals. We published four consecutive studies on publishers, number of articles, indicators, APC, list price, and OA status using JCR journals in the Science Citation Index Expanded (SCIE) and Social Sciences Citation Index (SSCI) [1-4]. JCR 2020, which was released in June 2021, expanded to include the Arts and Humanities Citation Index and the Emerging Sources Citation Index, but the impact factors (IFs) and percentage of OA gold were not yet presented for those resources. The inclusion of information on the percentage of OA gold for the past 3 years' ratio of OA articles in WoS made it easier to understand OA trends. Thus, it would be meaningful to analyze and compare the changes in journal publishing by year or by publisher type, including mega journals, based on JCR.

Knowledge of the environmental changes in journal publishing is useful for librarians who are considering economic subscription contracts and reasonable user services. For an accurate analysis of the rapidly changing and diverse journal environment, ongoing research based on reliable journal data is required. JCR lists only peer-reviewed journals through a rigorous evaluation process; therefore, an analysis of publication trends focusing on major journals that have been consistently listed in JCR would enable more accurate predictions of future changes in the journal publishing market.

Objectives: The purpose of this study was to analyze how the traditional journal publishing market, which was previously concentrated on peer-reviewed subscription journals produced by large commercial publishers, is changing in terms of article share and growth by publisher and access type with the expansion of $\mathrm{OA}$ journals and articles. Its results, with precise and up-to-date data, will help journal stakeholders establish journal subscription plans and APC support policies.

\section{Methods}

Ethics statement: This was not a study with human subjects, so neither institutional review board approval nor informed consent was required.

Study design: This was a literature database-based descriptive study.

Data collection and analysis: We used JCR instead of the journals in DOAJ and Scopus to identify the recent publication trends of only major journals. Research data were collected from JCR 2016, 2018, and 2020-2 years before and after JCR 2018, which began to have information on the percentage of OA gold-and an analysis was conducted by integrating the JCR journal lists into a single Excel file, similar to previous studies [1-4]. As of July 2021, SCIE and SSCI data of JCR 2016, 2018, and 2020 were downloaded as a text file and converted into an Excel file. The same journals were integrated using journal names and International Standard Serial Number, and affiliated publishers were classified according to the holding company (the recently merged BMC [BioMed Central] was classified as separate from Springer). Articles and reviews, which are counted as citable items in JCR, were considered as the number of articles per journal; thus, only journals with at least one citable item were identified as JCR journals, excluding journals with no citable items.

Among the collected data, major journals that were consistently listed in JCR 2016, 2018, and 2020 were extracted and pivot tables were used to calculate the compound annual growth rate (CAGR). In order to investigate changes by publisher type, 19 publishers that published more than 10,000 articles per year on average were selected and classified into four types. In addition to the current status of OA journals, trends in mega journals (defined as those containing more than 1,000 articles per year) were also analyzed.

Statistical methods: Data were tabulated and the proportions of the cells were calculated by pivot tables. The growth rate (\%) was calculated in terms of the CAGR.

\section{Results}

\section{JCR journals and articles}

JCR journals were grouped into major journals, which were consistently listed in JCR 2016, 2018, and 2020, and minor journals, which were listed only once or twice in the 3 editions of JCR due to new entry or exclusion. The former can be regarded as core journals with higher usability for researchers 
Table 1. Journals and articles in JCR

\begin{tabular}{|c|c|c|c|c|c|}
\hline & JCR 2016 & $\operatorname{CAGR}(\%)$ & JCR 2018 & CAGR (\%) & JCR 2020 \\
\hline Journals & 363 & 50.6 & 823 & 22.4 & 1,234 \\
\hline Articles & 41,827 & 22.2 & 62,465 & 71.8 & 184,269 \\
\hline \multicolumn{6}{|c|}{ Major journals ${ }^{b /}$} \\
\hline Journals & 10,953 & - & 10,953 & - & 10,953 \\
\hline Citations & $57,626,438$ & 9.8 & $69,424,170$ & 15.5 & $92,686,632$ \\
\hline Average IF & 2.228 & 6.1 & 2.510 & 15.0 & 3.322 \\
\hline Average II & 0.532 & 14.5 & 0.698 & 30.2 & 1.183 \\
\hline \multicolumn{6}{|l|}{ All journals } \\
\hline Average IF & 2.180 & 6.8 & 2.487 & 16.5 & 3.377 \\
\hline Average ॥ & 0.526 & 14.8 & 0.693 & 31.3 & 1.194 \\
\hline
\end{tabular}

JCR, Journal Citation Reports; CAGR, compound annual growth rate; IF, impact factor; II, immediacy index.

a) Journals listed once or twice in JCR 2016, 2018, and 2020; b) Journals consistently listed in JCR 2016, 2018, and 2020.

than the latter. Table 1 shows the analysis results of 10,953 major journals and minor journals. The number of major journals to all JCR journals decreased from $96.8 \%$ to $93.0 \%$ and $89.9 \%$, implying that the journals newly entering JCR increased gradually. Among the JCR articles, $94.7 \%$ were published in major journals and only $5.3 \%$ in minor journals.

As about $95 \%$ of JCR articles were published in major journals, their CAGR was very similar to that of the entire JCR. From JCR 2018 to JCR 2020, the CAGR for the increase of journals was only $1.7 \%$, but the CAGR for articles was $13.5 \%$, reaching the first year with over 2 million articles. The CAGRs for articles and citations were much higher in JCR 2020 than in JCR 2018. The CAGRs for average IF and the immediacy index were more than doubled, and each value entered the 3-point and 1-point range for the first time in JCR 2020. The average IF increased at a similar CAGR to that of citations, but the average immediacy index increased about twice as much as the average IF. These findings indicate that there have been significant changes in article publication and use.

\section{Major journals in JCR}

In JCR 2020, the OA data show the gold OA materials published in 2018, 2019, and 2020, and citations in 2020 to these items. To classify journal types according to the percentage of OA gold, gold OA journals were defined as those with a percentage of $95 \%$ and above, hybrid journals as those with a percentage of less than $95 \%$, and subscription-only journals as those with a percentage of $0 \%$. The status (Table 2) of OA journals and articles in JCR major journals was different from that reported in a previous study [3], which directly analyzed OA articles by year. Looking at only 10,953 major journals, the number of subscription-only journals decreased significantly in JCR 2020 compared to JCR 2018. Meanwhile, the number of hybrid and gold OA journals increased, with a higher proportion of hybrid journals than gold OA journals.

Fig. 1 shows the status of articles in the JCR major journals. In JCR 2020, the growth rate of total articles was over $10 \%$, which resulted from a significant increase in OA articles $(22.6 \%)$ along with the natural increase in non-OA articles (7.3\%). The share of OA articles in major journals in JCR 2020 was estimated to be $26.0 \%$.

\section{JCR major journals by publisher type}

It was assumed that there were many changes in JCR journals and publishers due to the radical growth in OA publications; therefore, the publishers of major journals were divided into 5 groups, and each group's articles and citations were compared (Table 3). There was little change in the total number of publishers of JCR journals, with a maximum of 2,082. Among the 19 publishers that published more than 10,000 articles per year, the status of large publishers was dominant, as in the previous study [1], while OA publishers grew rapidly over time. As shown in Fig. 2, six large publishers (including Elsevier) gradually increased their article and citation share to 
Table 2. Journals and articles in JCR major journals by OA type

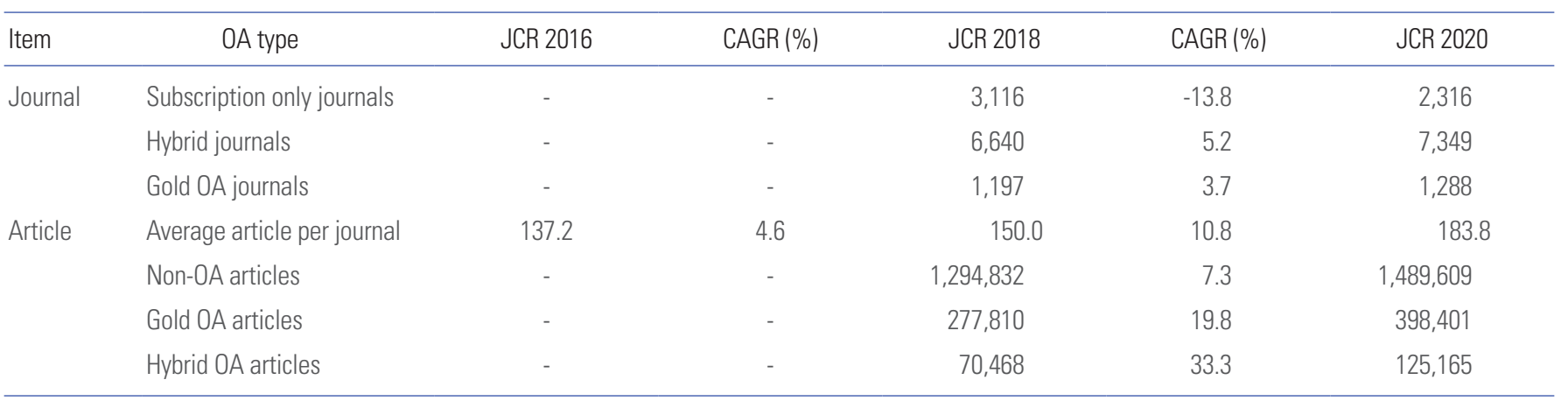

JCR provides OA information about gold OA articles since JCR 2018; Gold OA journals were defined as those with an OA gold percentage of 95\% and above, and hybrid OA journals as those with a percentage of less than $95 \%$.

JCR, Journal Citation Reports; OA, open access; CAGR, compound annual growth rate.

\begin{tabular}{|c|c|c|c|c|}
\hline JCR 2016 & CAGR (\%) & JCR 2018 & CAGR (\%) & JCR 2020 \\
\hline 1,502,324 Articles & 4.6 & $1,643,110$ Articles & 10.7 & 2,013,175 Articles \\
\hline $\begin{array}{l}\text { Non-OA articles } \\
\text { (no data) }\end{array}$ & - & $\begin{array}{c}\text { 1,294,832 Non-OA articles } \\
(78.8 \%)\end{array}$ & 7.3 & $\begin{array}{c}\text { 1,489,609 Non-0A articles } \\
(74.0 \%)\end{array}$ \\
\hline $\begin{array}{l}\text { OA articles } \\
\text { (no data) }\end{array}$ & - & $\begin{array}{c}348,278 \text { 0A articles } \\
(21.2 \%)\end{array}$ & 22.6 & $\begin{array}{c}523,5660 \mathrm{~A} \text { articles } \\
(26.0 \%)\end{array}$ \\
\hline
\end{tabular}

Fig. 1. Articles in major Journal Citation Reports (JCR) journals. CAGR, compound annual growth rate; OA, open access.

nearly $60 \%$. OA publishers grew so much that MDPI ranked fifth in article share, overtaking Sage, but their citation share was less than half of their article share. For the other publishers, both article and citation shares gradually decreased despite their growing CAGR.

\section{Distribution of JCR mega journals}

Table 4 shows the article distribution of the JCR major journals. Most journals (over 45\%) published 50-199 articles per year. About one-third of journals published fewer than 50 articles, while 20\% published over 200 articles. The number of journals in the former category decreased over time, while the number of journals in the latter category increased. Of particular note, mega journals (publishing over 1,000 articles per year) showed radical growth, with a CAGR of $11.6 \%$ in JCR 2018 and $15.6 \%$ in JCR 2020. The article share of the 231 mega journals in JCR 2020 was $26.5 \%$, and more than half
(132 journals) were influential journals, ranked in Journal Impact Factor quartile 1. In an analysis of the 231 mega journals by publisher type, 113 journals were distributed by large publishers, 49 journals by OA publishers, and 46 journals by society publishers. Elsevier was the largest individual publisher, publishing 73 mega journals, followed by MDPI, which published 25 mega journals. Among the 132 most influential mega journals, 104 journals were hybrid journals (i.e., subscription journals with numerous OA articles).

\section{Discussion}

Major changes in articles and citations

The CAGR of articles in major journals increased to $10.7 \%$ in JCR 2020, reflecting a more than 2 -fold increase since JCR 2018. In JCR 2020, there was no significant change in the number of publishers and journals, but it was confirmed that 
Table 3. JCR major journals by publisher type

\begin{tabular}{|c|c|c|c|c|c|c|}
\hline Publisher type & Item & JCR 2016 & CAGR (\%) & JCR 2018 & CAGR $(\%)$ & JCR 2020 \\
\hline \multirow[t]{2}{*}{ Large $^{\text {a) }}$ (6 publishers, 6,302 journals) } & Article & 859,248 & 4.4 & 936,507 & 13.1 & $1,198,997$ \\
\hline & Citation & $32,469,800$ & 10.4 & $39,571,698$ & 17.0 & $54,202,383$ \\
\hline \multirow[t]{2}{*}{$O A^{b)}$ (5 publishers, 292 journals) } & Article & 90,263 & 20.3 & 130,718 & 23.7 & 200,020 \\
\hline & Citation & $1,664,335$ & 21.3 & $2,448,625$ & 31.1 & $4,211,214$ \\
\hline \multirow[t]{2}{*}{ Society ${ }^{c)}$ (4 publishers, 250 journals) } & Article & 133,848 & 3.1 & 142,335 & 7.4 & 164,161 \\
\hline & Citation & $6,466,163$ & 9.7 & $7,782,579$ & 10.4 & $9,485,091$ \\
\hline \multirow[t]{2}{*}{ University \& other ${ }^{\mathrm{d})}$ (4 publishers, 557 journals) } & Article & 83,861 & 1.2 & 85,807 & 1.6 & 88,606 \\
\hline & Citation & $4,287,971$ & 6.9 & 4,899,138 & 10.5 & $5,978,032$ \\
\hline \multirow[t]{2}{*}{ The others ${ }^{\mathrm{e})}$ (1,761 publishers, 3,552 journals) } & Article & 335,104 & 1.9 & 347,743 & 1.9 & 361,391 \\
\hline & Citation & $12,738,169$ & 7.5 & $14,722,130$ & 13.0 & $18,809,912$ \\
\hline
\end{tabular}

JCR, Journal Citation Reports; CAGR, compound annual growth rate; OA, open access.

alElsevier, Springer, Wiley, Taylor \& Francis, Sage, Wolters Kluwer; ${ }^{b /}$ MDPI, Frontiers, Public Library of Science, Hindawi, BMC; ${ }^{\text {cl} A m e r i c a n ~ C h e m i c a l ~ S o c i e t y, ~ R o y a l ~}$ Society of Chemistry, Institute of Electrical and Electronics Engineers, American Physical Society; ${ }^{d}$ Oxford University Press, IOP Publishing, Cambridge University

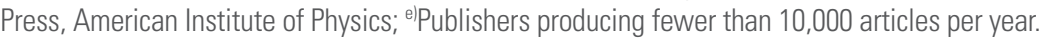

Table 4. Distribution of journals by number of annual articles

\begin{tabular}{|c|c|c|c|c|c|}
\hline No. of articles & $\begin{array}{c}\text { Journal in JCR } 2016 \\
\text { (journal share/article share) }\end{array}$ & CAGR (\%) & $\begin{array}{c}\text { Journal in JCR } 2018 \\
\text { (journal share/article share) }\end{array}$ & CAGR (\%) & $\begin{array}{c}\text { Journal in JCR } 2020 \\
\text { (journal share/article share) }\end{array}$ \\
\hline$<50$ & $4,183(38.2 / 8.2)$ & 2.1 & $4,013(36.6 / 7.3)$ & 10.1 & $3,243(29.6 / 4.7)$ \\
\hline 50-99 & $2,901(26.5 / 13.7)$ & -0.9 & $2,847(26.0 / 12.4)$ & 0.4 & $2,870(26.2 / 10.2)$ \\
\hline 100-199 & $2,108(19.2 / 19.5)$ & 1.2 & 2,158 (19.7/18.3) & 5.2 & 2,389 (21.8/16.8) \\
\hline 200-299 & $803(7.3 / 13.0)$ & 2.5 & $844(7.7 / 12.5)$ & 9.6 & $1,014(9.3 / 12.3)$ \\
\hline 300-499 & $542(4.9 / 13.6)$ & 3.8 & $584(5.3 / 13.4)$ & 14.1 & $760(6.9 / 14.4)$ \\
\hline 500-999 & $277(2.5 / 12.7)$ & 9.8 & 334 (3.0/13.6) & 15.6 & $446(4.1 / 15.0)$ \\
\hline$\geq 1,000$ & $139(1.3 / 19.4)$ & 11.6 & $173(1.6 / 22.5)$ & 15.6 & $231(2.1 / 26.5)$ \\
\hline
\end{tabular}

JCR, Journal Citation Reports; CAGR, compound annual growth rate.

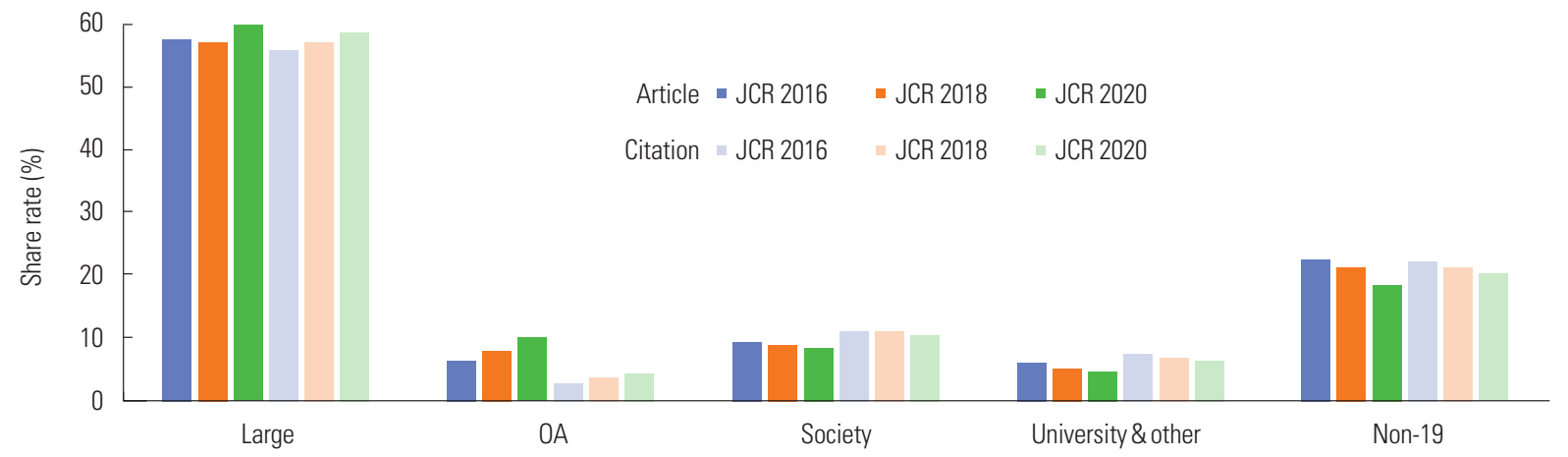

Fig. 2. Article and citation shares by publisher type. JCR, Journal Citation Reports; OA, open access.

the average IF and immediacy index increased rapidly, along with the increase of articles and citations. In particular, the CAGR of the average immediacy index was double than that of the average IF, which is assumed to be due to the increase of OA articles, early promotion of new articles, and free articles for temporary services, which resulted in faster access to articles by users. However, further studies are needed to find the exact cause. In an analysis according to publisher type, 
large publishers continued to increase their article and citation shares to nearly $60 \%$, while the remaining publishers (except OA publishers) maintained a citation share higher than their article share, even though both decreased. These changes in the share of articles and citations by publisher type will be referred to in journal package contracts.

\section{Implications for the increase of $\mathrm{OA}$ journals and articles}

As $\mathrm{OA}$ articles are welcomed by libraries and researchers, the growing influence of $\mathrm{OA}$ journals and articles with the rapid progress of OA publishing merits attention. In JCR 2020, the CAGR of OA articles was 3 times higher than that of non-OA articles, and the share of OA articles in JCR major journals reached $26.0 \%$. Although this share is lower than that of $27.1 \%$ [3] for all journals in JCR 2019, which directly reflected the number of OA articles in WoS by year, it was found to be higher than has been reported in other studies (the share of OA articles in 2018 was $18.9 \%$ in Scopus [5], and $24.0 \%$ in WoS [6]). Hybrid OA articles increased at a higher rate than gold $\mathrm{OA}$ articles, but gold $\mathrm{OA}$ articles were much more numerous. In a previous study [3], some gold OA journals showed superior influence in several JCR indicators. However, in JCR major journals, $\mathrm{OA}$ articles issued by $\mathrm{OA}$ publishers increased with the highest CAGR, but their citation share was less than one-half of their article share. It is assumed that there are substantial differences in influence among OA journals, and institutions need to reflect these differences when supporting APC fees for each journal.

Mega journals as major changers of the journal market In order to forecast the future journal market, the influence of mega journals is noteworthy. In JCR 2020, mega journals made a substantial contribution to the article share (26.5\%), with numerous OA articles, despite their small journal share (2.1\%), and showed a high level of excellence in terms of their Journal Impact Factor quartile. When mega journals appeared, they were embroiled in controversy over predatory journals, as they were initiated by new OA publishers. However, in JCR 2020 , traditional print journal publishers actively participated in publishing mega journals, as six large publishers accounted for about 50\% (including Elsevier's 32\%) and four society publishers accounted for $20 \%$ of the 231 mega journals. Moreover, among the 132 excellent mega journals (in Journal Impact Factor quartile 1), 79\% were hybrid journals. This means that mega journals were no longer limited to OA publishers; instead, traditional print journal publishers were at the forefront of publishing mega journals. The results of this study suggest that the influence of mega journals is expected to grow greatly; therefore, journal stakeholders should pay attention to mega journals as a substantial source of changes in the future journal market. Due to recent changes, wherein article submission and use are driven to these mega journals, a new evaluation of mega journals (including OA journals) is required.

Continuing increases in journal subscription fees

To find reasons for increases in journal subscription fees, only subscription articles were considered (i.e., with the exclusion of gold and hybrid OA articles). Regarding the issue of journal subscription fees, one researcher pointed out the high profits of the major commercial publishers, concentrated contracts for "big deal" electronic licenses, high barriers to new entrants, and protected competitive positions for industry rivalries [7]. Non-OA articles increased by 7.3\% in JCR 2020 and became an important factor for publishers to raise journal subscription fees. While six large publishers maintained a high share (nearly $60 \%$ ) in articles, their growth rate $(13.1 \%)$, which was higher than the overall rate (10.7\%), may also have been an important factor explaining why large publishers demanded higher increases in journal subscription fees. Despite the high growth of OA articles, the natural growth of both non-OA articles and total articles, which was higher than the inflation rate, continues to make it difficult to negotiate subscription fees between libraries and publishers. However, a study on reductions in publication cost with journal volume reported that with a rejection rate of around $90 \%$, publishing costs are 1,054 US dollars for 100 articles or 771 US dollars for 1,000 articles [8]. This suggests that a new strategy can be considered for negotiating subscription fees depending on how many hybrid journals with numerous $\mathrm{OA}$ articles are included in the journal packages.

Limitations: The analytical results of this study reflect the assumption that the WoS journals in 2021 are identical to major journals in JCR, without changes in the publisher. In addition, the number of OA articles was easily estimated by using JCR's percentage of OA gold, but caution is needed as there was a slight difference from the previous study [3] in how OA articles were calculated and gold OA journals were determined. A regrettable limitation is that the correlation analysis between the markedly increased average immediacy index and IF was excluded from the scope of this study.

Conclusion: This study analyzed the journal publishing environment, focusing on major journals that were consistently listed in JCR and publishing about $95 \%$ of articles. Among the 19 publishers publishing more than 10,000 articles per year, in JCR 2020, six large publishers accounted for $59.6 \%$ of articles and the remaining 13 publishers for $22.5 \%$, with the other publishers (about 2,000 publishers not included in the 19 publishers) for $17.9 \%$. Under the strong influence of large publishers, OA publishers grew rapidly, but the share of the remain- 


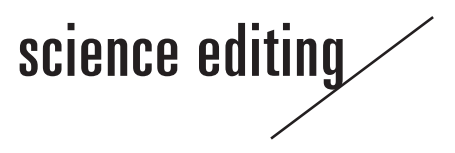

ing publishers gradually decreased. Even though the growth rate of OA articles was higher than that of non-OA articles, the natural growth of non-OA articles and total articles was assumed to be main factor leading publishers to raise journal subscription fees. The growth rate of the average immediacy index was nearly double than that of the average IF, implying that major changes may have been taking place in journal publishing and the research community. Large and society publishers, rooted in their experiences with traditional print journals, published mega journals actively, along with OA publishers. Furthermore, mega journals showed a strong influence on the increase of articles and citations. Journal stakeholders need to continue to pay attention to these changes. This study is expected to be useful for libraries in negotiating journal subscription fees with publishers, planning journal subscription, and supporting APC fees for OA articles.

\section{Conflict of Interest}

No potential conflict of interest relevant to this article was reported.

\section{Funding}

The authors received no financial support for this article.

\section{Data Availability}

Most of the raw data in this paper are various indicators of JCR, which is sold as a paid commercial database; therefore, sharing is not available. Please contact the corresponding author for raw data availability.

\section{References}

1. Kim SJ, Park KS. Influence of the top 10 journal publishers listed in Journal Citation Reports based on six indicators. Sci Ed 2020;7:142-8. https://doi.org/10.6087/kcse.209

2. Kim SJ, Park KS. Market share of the largest publishers in Journal Citation Reports based on journal price and article processing charge. Sci Ed 2020;7:149-55. https://doi.org/ $10.6087 / \mathrm{kcse} .210$

3. Kim SJ, Park KS. Open access status of journals and articles in Journal Citation Reports. Sci Ed 2021;8:26-31. https:// doi.org/10.6087/kcse.226

4. Kim SJ, Park KS. Influence of open access journals on the research community in Journal Citation Reports. Sci Ed 2021;8:32-8. https://doi.org/10.6087/kcse.227

5. Bjork BC, Korkeamaki T. Adoption of the open access business model in scientific journal publishing: a cross-disciplinary study. Coll Res Libr 2020;81:1080-94. https://doi. org/10.5860/crl.81.7.1080

6. AlRyalat SA, Saleh M, Alaqraa M, et al. The impact of the open-access status on journal indices: a review of medical journals [version 1]. F1000Research [Preprint]. 2019;8:266 [cited 2020 Sep 29]. https://doi.org/10.12688/f1000research. 17979.1

7. Bjork BC. Why is access to the scholarly journal literature so expensive?. Portal Libr Acad 2021;21:177-92. https://doi. org/10.1353/pla.2021.0010

8. Grossmann A, Brembs B. Current market rates for scholarly publishing services [version 2]. F1000Research [Preprint]. 2021;10:20 [cited 2021 Sep 27]. https://doi.org/10.12688/ f1000research. 27468.2 